\title{
WHAT RANDOM VARIABLE GENERATES A BOUNDED POTENTIAL?
}

\author{
N. KARTASHOV AND YU. MISHURA
}

Received 29 March 2002 and in revised form 4 October 2003

It is known that if a predictable nondecreasing process generates a bounded potential, then its final value satisfies the Garsia inequality. We prove the converse, that is, a random variable satisfying the Garsia inequality generates a bounded potential. We also propose some useful relations between the Garsia inequality and the Cramer conditions, and different ways how to construct a potential.

\section{Introduction}

Let $(\Omega, \mathscr{F}, \mathbb{P})$ be a complete probability space and let $\left\{\mathscr{F}_{t}, t \geq 0\right\}$ be a filtration satisfying the standard conditions: $\mathscr{F}_{0}$ contains $\mathbb{P}$ zero sets of $\sigma$-field $\mathscr{F}_{,}, \mathscr{F}_{s} \subset \mathscr{F}_{t} \subset \mathscr{F}, 0 \leq s \leq t$, $\mathscr{F}_{s}=\bigcap_{t>s} \mathscr{F}_{t}$. Also, let $\left\{X_{t}, \mathscr{F}_{t}, t>0\right\}$ be the potential with the Doob-Meyer decomposition of the form

$$
X_{t}=M_{t}-A_{t}
$$

where $M_{t}$ is a martingale, $A_{t}$ is nondecreasing integrable right-continuous predictable process, $A_{0}=0$. Let $A_{\infty}:=\lim _{t \rightarrow \infty} A_{t}$. It follows from (1.1) that $X_{t}$ also admits the decomposition

$$
X_{t}=E\left(A_{\infty}-A_{t} \mid \mathscr{F}_{t}\right),
$$

and we say that the process $A_{t}$ generates the potential $X_{t}$. Garsia [2] established that in the case when $X_{t}$ is bounded, that is, $0 \leq X_{t} \leq c_{0}$, the random variable (r.v.) $\xi:=A_{\infty}$ satisfies the Garsia inequality

$$
E G(\xi) \leq c_{0} E g(\xi)
$$

where $g=g(t): \mathbb{R}_{+} \rightarrow \mathbb{R}_{+}$is any nondecreasing nonnegative function, $G(t)=\int_{0}^{t} g(u) d u$. In particular, with $g(t)=t^{n-1}, n \geq 1$, we obtain from the Garsia inequality that $\xi$ satisfies the Cramer conditions, that is, $E \xi^{n} \leq c_{0}^{n} \cdot n !, n \geq 1$. 
Our aim is to investigate what sort of potential can be generated by a r.v. that satisfies the Garsia inequality or the Cramer conditions.

The paper is organized as follows. Section 2 contains relations between the Garsia inequality and the Cramer conditions on the one side, and the properties of the distribution function (d.f.) of corresponding r.v. on the other side. Example 2.6 demonstrates that the Garsia inequality and the Cramer conditions are not equivalent. In Section 3 we prove that any r.v. satisfying the Garsia inequality predictably generates a nontrivial bounded potential (corresponding definitions are provided in this section). In Section 4 we transform some r.v. in such a way that the resulting r.v. predictably generates a bounded potential.

\section{The asymptotic properties of a distribution function corresponding to a random variable that satisfies the Garsia inequality or the Cramer conditions}

Throughout the paper, $\xi$ is a nonnegative unbounded integrable r.v. with the d.f. $F_{\xi}(x)=$ $\mathbb{P}\{\xi<x\}$. Note that the integral $\int_{x}^{\infty}\left(1-F_{\xi}(u)\right) d u$ exists and is positive for any $x \geq 0, E \xi=$ $\int_{0}^{\infty}\left(1-F_{\xi}(u)\right) d u$, and $1-F_{\xi}(x)$ is a left-continuous function.

Denote

$$
\lambda(x):=\left(1-F_{\xi}(x)\right)\left(\int_{x}^{\infty}\left(1-F_{\xi}(u)\right) d u\right)^{-1}=(E(\xi-x \mid \xi \geq x))^{-1} .
$$

Lemma 2.1. (1) The positive, left-continuous function $\lambda$ defined by (2.1) also satisfies the relations

$$
\begin{aligned}
& 1-F_{\xi}(x)=E \xi \cdot \lambda(x) \exp \left\{-\int_{0}^{x} \lambda(u) d u\right\} \\
& \int_{0}^{x} \lambda(u) d u=-\ln \left(\int_{x}^{\infty}\left(1-F_{\xi}(u)\right) d u \cdot(E \xi)^{-1}\right), \\
& \int_{0}^{\infty} \lambda(u) d u=\infty
\end{aligned}
$$

(2) Let the function $\lambda$ satisfy representation (2.2). Then it is unique, positive, left-continuous, and satisfies (2.1), (2.3), and (2.4).

Proof. (1) Let $\lambda$ be defined by (2.1). Denote $Q(x):=\int_{x}^{\infty}\left(1-F_{\xi}(u)\right) d u$. Then $\int_{0}^{x} \lambda(u) d u=$ $\int_{Q(x)}^{Q(0)}(d u / u)=\ln (Q(0) / Q(x))$, yielding $(2.3)$ and also $E \xi \cdot \lambda(x) \exp \left\{-\int_{0}^{x} \lambda(u) d u\right\}=E \xi$. $\lambda(x) \cdot(Q(x) / Q(0))=\lambda(x) Q(x)=1-F_{\xi}(x)$, that is (2.2). Evidently, $Q(\infty)=0$ and therefore $\int_{0}^{\infty} \lambda(u) d u=\ln (Q(0) / 0)=\infty$.

(2) Let the function $\lambda$ be defined by (2.2). Denote $\Lambda(x):=\int_{0}^{x} \lambda(u) d u$, and obtain from (2.2) that

$$
\begin{aligned}
\int_{0}^{x}\left(1-F_{\xi}(u) d u\right) & =E \xi \cdot \int_{0}^{x} \exp \{-\Lambda(u)\} \lambda(u) d u \\
& =E \xi \cdot(1-\exp \{-\Lambda(x)\}) .
\end{aligned}
$$


Since $E \xi=\int_{0}^{\infty}\left(1-F_{\xi}(u)\right) d u$, we obtain from (2.5) that $E \xi \cdot \exp \{-\Lambda(x)\}=\int_{x}^{\infty}(1-$ $\left.F_{\xi}(u)\right) d u$. Therefore, from (2.2),

$$
1-F_{\xi}(x)=\lambda(x) \cdot \int_{x}^{\infty}\left(1-F_{\xi}(u)\right) d u
$$

and, also, $E \xi \exp \{-\Lambda(\infty)\}=0$. Hence $\Lambda(\infty)=\infty$. We establish (2.1) and (2.4). Relation (2.3) follows from (2.1) (see part (1) of the proof).

THeOREM 2.2. Let $\xi$ be a nonnegative unbounded integrable r.v. The following assertions are equivalent:

(i) r.v. $\xi$ satisfies Garsia inequality (1.3);

(ii) for any $x \geq 0, \lambda(x) \geq c_{0}^{-1}$, where $\lambda$ is defined by (2.1).

Remark 2.3. It follows from (2.1) that (ii) is equivalent to

(iii) $\sup _{x \geq 0} E(\xi-x \mid \xi \geq x) \leq c_{0}$.

Proof. (1) Let r.v. $\xi$ satisfy Garsia inequality (1.3). Then, in particular, $E \xi \leq c_{0}$. Consider a nonnegative nondecreasing function $g$ such that $E g(\xi)<\infty$. Then,

$$
\begin{aligned}
0 \leq & c_{0} E g(\xi)-E G(\xi) \\
= & c_{0} \int_{0}^{\infty}\left(g(0)+\int_{0+}^{u} d g(v)\right) F_{\xi}(d u) \\
& -\int_{0}^{\infty} \int_{0}^{u}\left(g(0)+\int_{0+}^{r} d g(v)\right) d r F_{\xi}(d u) \\
= & \left(c_{0}-E \xi\right) g(0)+\int_{0+}^{\infty}\left(c_{0}\left(1-F_{\xi}(u)\right)-\int_{u}^{\infty}\left(1-F_{\xi}(v)\right) d v\right) d g(u) .
\end{aligned}
$$

The integrand on the right-hand side of (2.7) is left-continuous. So, if (2.7) holds for any nonnegative nondecreasing $g$ with $\operatorname{Eg}(\xi) \leq \infty$, then obviously this integrand is nonnegative, that is,

$$
c_{0}\left(1-F_{\xi}(u)\right)-\int_{u}^{\infty}\left(1-F_{\xi}(v)\right) d v \geq 0 \quad \text { for each } u \geq 0 .
$$

Inequality (2.8), by Lemma 2.1 , is equivalent to $\lambda(u) \geq c_{0}^{-1}, u \geq 0$.

(2) Let $\lambda(x) \geq c_{0}^{-1}, x \geq 0$. From $\lambda(0)=(E \xi)^{-1}$, we obtain $E \xi \leq c_{0}$. From the latter and relation (2.1), we deduce that the right-hand side of (2.7) is nonnegative for any nonnegative nondecreasing $g$, and for such $g$ with $E g(\xi)<\infty$, it equals $c_{0} E g(\xi)-E G(\xi)$. Thus we arrive at the Garsia inequality.

Remark 2.4. If we consider $F_{\xi}(x)=\mathbb{P}\{\xi \leq x\}$, that is, right-continuous $F_{\xi}(x)$, and $\xi$ has no atom at zero, the proof of Theorem 2.2 will be the same. But if $\xi$ has an atom at zero and $F_{\xi}(x)$ is right-continuous, then we must consider $\lambda(x)$ with some additional term, 
namely,

$$
\begin{aligned}
\lambda(x) & :=\left(1-F_{\xi}(x)+P\{\xi=0\} I\{x=0\}\right)\left(\int_{x}^{\infty}\left(1-F_{\xi}(u)\right) d u\right)^{-1} \\
& =(E(\xi-x \mid \xi \geq x))^{-1} .
\end{aligned}
$$

To avoid these technical difficulties, we therefore consider left-continuous functions.

Theorem 2.5. Let $\xi$ be the same as in Theorem 2.2. The following assertions are equivalent:

(iv) the r.v. $\xi$ satisfies the Cramer conditions;

(v) $\underline{\lim }_{x \rightarrow \infty}(1 / x) \int_{0}^{x} \lambda(u) d u>0$, where $\lambda$ is defined by (2.1).

Proof. It is well known (see [3]) that the Cramer conditions hold if and only if there exists an $r>0$ such that $E \exp \{r \xi\}<\infty$. In turn, the last inequality is equivalent to

$$
1-F_{\xi}(x) \leq c \exp \{-r x\}, \quad x \geq 0
$$

for some $0<r<\infty$ and $0<c<\infty$, since $E \exp \{r \xi\} \geq \int_{x}^{\infty} \exp \{r u\} d F_{\xi}(u) \geq \exp \{r x\}(1-$ $\left.F_{\xi}(x)\right)$, and $E \exp \{\bar{r} \xi\}=1+\bar{r} \int_{0}^{\infty}\left(1-F_{\xi}(u)\right) \exp \{\bar{r} u\} d u, \bar{r} \in(0, r)$.

By the same arguments, we obtain that $(2.10)$ is equivalent to

$$
\int_{x}^{\infty}\left(1-F_{\xi}(u)\right) d u \leq c \exp \{-r x\}, \quad x \geq 0
$$

for some $0<r<\infty$ and $0<c<\infty$. From relation (2.3), we deduce that the Cramer conditions are equivalent to the inequality

$$
\int_{0}^{x} \lambda(u) d u \geq r x+c_{1}, \quad x \geq 0
$$

for some $r>0, c_{1} \in \mathbb{R}$, that is, equivalent to (v).

As mentioned above, the Cramer conditions follow from the Garsia inequality. The next example demonstrates that the converse is not true.

Example 2.6. There exists an integrable nonnegative r.v. $\xi$ satisfying the Cramer conditions but not the Garsia inequality. The idea is the following. If we choose a nonnegative left-continuous function $\lambda(x)$ such that $\ln \lambda(x)-\int_{0}^{x} \lambda(u) d u$ decreases to $-\infty$, and $\int_{0}^{x} \lambda(u) d u \geq r x+c_{1}, x \geq 0$, for some $r>0, c_{1} \in \mathbb{R}$, but $\underline{\lim }_{x \rightarrow \infty} \lambda(x)=0$, then the righthand side of (2.2) will be a tail of some d.f. $F_{\xi}(x)$. This d.f. will satisfy the Cramer conditions by Theorem 2.5, but the Garsia inequality will not hold by assumption (ii) of Theorem 2.2.

Three conditions mentioned above will be satisfied if the function $\lambda$ is a solution of the boundary value problem

$$
-\lambda^{\prime}(x)+\lambda^{2}(x)=\lambda(x) \varphi(x), \quad x \geq 0, \quad \lambda(0)=\frac{1}{3},
$$

where $\varphi(x)=\sum_{n \geq 0} I_{[2 n, 2 n+1)}(x)$. 
The corresponding d.f. $F_{\xi}(x)$ can be calculated from (2.2) as

$$
\begin{aligned}
1-F_{\xi}(x) & =E \xi \cdot \lambda(0) \cdot \exp \left\{\int_{0+}^{x}(d \ln \lambda(y)-\lambda(y) d y)\right\} \\
& =\frac{1}{2} E \xi \exp \left\{-\int_{0}^{x} \varphi(y) d y\right\} \sim c \exp \left\{-\frac{1}{2} x\right\}
\end{aligned}
$$

whence the Cramer conditions hold.

The solution of (2.13) on the intervals $[2 n, 2 n+1)$ and $[2 n+1,2 n+2)$ equals

$$
\lambda(x)=\left\{\begin{array}{l}
\lambda(2 n) e^{2 n}\left(\lambda(2 n) e^{2 n}+(1-\lambda(2 n)) e^{x}\right)^{-1}, \quad x \in[2 n, 2 n+1), \\
\lambda(2 n+1)((2 n+1) \cdot \lambda(2 n+1)+1-\lambda(2 n+1) x)^{-1}, \quad x \in[2 n+1,2 n+2),
\end{array}\right.
$$

supposing that $\lambda \in C\left(\mathbb{R}_{+}\right)$. Evidently, $\lambda(x)$ increases on $[2 n+1,2 n+2)$, and decreases on $[2 n, 2 n+1), n \geq 0$. The values of $\lambda(2 n+1)$ at the points $x_{n}=2 n+1$ of a local minimum can be estimated from the relations $\lambda(1)=(1+2 e)^{-1}<1 / 6 ; \lambda(2 n+1)=\left(1+\left((\lambda(2 n))^{-1}-\right.\right.$ $1) e)^{-1}, \lambda(2 n)=\lambda(2 n-1)(1-\lambda(2 n-1))^{-1}$, whence $\lambda(2 n+1)=\left(1+\left((\lambda(2 n-1))^{-1}-\right.\right.$ $2) e)^{-1}$. Suppose that $\lambda(2 n-1)<1 /(n+5)$ (it is true for $\left.n=1\right)$, then $\lambda(2 n+1)<(1+(n+$ $3) e)^{-1}<1 /(n+6)$. It means that $\lambda(2 n+1) \rightarrow 0, n \rightarrow \infty$, and $\underline{\lim }_{x \rightarrow \infty} \lambda(x) \leq \underline{\lim }_{n \rightarrow \infty} \lambda(2 n+$ $1)=0$, so the Garsia inequality does not hold.

Theorem 2.7. Let nonnegative unbounded r.v. $\xi$ satisfy the Cramer conditions. Then $\overline{\lim }_{x \rightarrow \infty} \lambda(x)>0$.

Proof. Choose an $r>0$ such that

$$
E \exp \{r \xi\}<\infty
$$

Then from (2.2) and Raikov theorem (see [3]), for any $0<\bar{r}<r,\left(1-F_{\xi}(x)\right) \exp \{\bar{r} x\} \rightarrow 0$, $x \rightarrow \infty$, and

$$
\begin{aligned}
E \exp \{\bar{r} \xi\} & =\bar{r} \int_{0}^{\infty} \exp \{\bar{r} x\}\left(1-F_{\xi}(x)\right) d x \\
& =\bar{r} \int_{0}^{\infty} \exp \left\{\bar{r} x-\int_{0}^{x} \lambda(u) d u\right\} \lambda(x) d x \cdot E \xi
\end{aligned}
$$

Suppose that $\lambda(x) \rightarrow 0, x \rightarrow \infty$. Choose any $\varepsilon \in(0, \bar{r})$. Then there exists $x_{0}>0$ such that $\lambda(x)<\varepsilon$, for $x>x_{0}$, whence

$$
\begin{aligned}
\int_{0}^{\infty} \exp & \left\{\bar{r} x-\int_{0}^{x} \lambda(u) d u\right\} \lambda(x) d x \\
& \geq \exp \left\{\int_{0}^{x_{0}}(r-\lambda(u)) d u\right\} \cdot \int_{0}^{\infty} \exp \left\{(r-\varepsilon)\left(x-x_{0}\right)\right\} \lambda(x) d x \\
& >\exp \left\{\int_{0}^{x_{0}}(r-\lambda(u)) d u\right\} \cdot \int_{0}^{\infty} \lambda(x) d x=\infty .
\end{aligned}
$$


Relations (2.17) and (2.18) contradict (2.16). So our assumption is false, whence the proof follows.

The next example demonstrates that the inequality $\varlimsup_{x \rightarrow \infty} \lambda(x)>0$ is not equivalent to the Cramer conditions.

Example 2.8. Denote

$$
\begin{aligned}
& x_{n}:=\frac{(n-1) n(2 n-1)}{6}+\frac{(n-1) n}{2}, \\
& y_{n}:=\frac{(n+1) n(2 n+1)}{6}+\frac{n(n-1)}{2}, \quad n \geq 1 .
\end{aligned}
$$

Let $\lambda(0)=1$,

$$
\lambda(x)= \begin{cases}\frac{1}{\sqrt{n}}, & x_{n}<x \leq y_{n} \\ \frac{1}{\sqrt{n}}+\frac{1}{n}\left(x-y_{n}\right), & y_{n} \leq x \leq x_{n+1}, n \geq 1 .\end{cases}
$$

Then $\varlimsup_{x \rightarrow \infty} \lambda(x) \geq \lim _{n \rightarrow \infty} \lambda\left(x_{n+1}\right)=\lim _{n \rightarrow \infty}(1+1 / \sqrt{n})=1$.

Denote $\psi(x):=\lambda^{\prime}(x)-\lambda^{2}(x)$. Then $\psi(x) \leq 1 / n-(1 / \sqrt{(n)})^{2}=0$ and $\lambda$ has only negative jumps. Therefore, $\lambda(x) \exp \left\{-\int_{0}^{x} \lambda(u) d u\right\}$ is nonincreasing. It means that $1-F_{\xi}(x)$ is considered as the left-hand side of (2.1), with $E \xi=1$ being nonincreasing. Moreover, for $x_{n}<x<x_{n+1}, n \geq 2$,

$$
\begin{aligned}
& \frac{1}{\sqrt{n}} \exp \left\{-\sum_{k=1}^{n}\left(\frac{1}{\sqrt{k}} k^{2}+\frac{k}{2}+\sqrt{k}\right)\right\} \\
& \quad \leq 1-F_{\xi}(x) \leq\left(\frac{1}{\sqrt{n}}+1\right) \exp \left\{-\sum_{k=1}^{n-1}\left(\frac{1}{\sqrt{k}} k^{2}+\frac{k}{2}+\sqrt{k}\right)\right\} .
\end{aligned}
$$

From the right-hand side of $(2.21), 1-F_{\xi}(x) \leq \exp \left\{-(2 / 5)(n-1)^{5 / 2}\right\}$, and from the lefthand side of (2.21),

$$
1-F_{\xi}(x)>\frac{1}{\sqrt{n}} \exp \left\{-\frac{6}{5}(n+1)^{5 / 2}\right\}, \quad x_{n}<x \leq x_{n+1} .
$$

Evidently, $x_{n}>(n-1)^{3} / 3, x_{n+1}<(5 / 6)(n+1)^{3}$. So, for sufficiently large $x$,

$$
1-F_{\xi}(x) \leq \exp \left\{-\frac{2}{5}\left(\left(\frac{6}{5} x\right)^{1 / 3}-2\right)^{5 / 2}\right\} \sim \exp \left\{-\alpha x^{5 / 6}\right\}
$$


for some constant $\alpha>0$. Therefore $\xi$ is integrable r.v. On the other hand,

$$
1-F_{\xi}(x)>\left((3 x)^{1 / 3}+1\right)^{-1 / 2} \exp \left\{-\frac{6}{5}\left((3 x)^{1 / 3}+2\right)^{5 / 2}\right\} \sim \beta x^{-1 / 6} \exp \left\{-\gamma x^{5 / 6}\right\}
$$

for some constants $\beta, \gamma>0$. It means that $E \exp \{r \xi\}=\infty$ for any $r>0$; so $\xi$ does not satisfy the Cramer conditions.

\section{What random variable generates a bounded potential?}

Definition 3.1. A r.v. $\xi$ is said to generate a bounded potential if there exists a filtration $\left\{G_{t}, t \geq 0\right\}$ on $(\Omega, \mathscr{F}, \mathbb{P})$ that satisfies the standard conditions, and a bounded rightcontinuous $G_{t}$-adapted potential $X_{t}$ that admits the expansion (compare with (1.2))

$$
X_{t}=E\left(A_{\infty}-A_{t} \mid G_{t}\right),
$$

with a $G_{t}$-adapted nondecreasing integrable process $A_{t}$ such that $A_{\infty}=\xi$.

Definition 3.1 means, in particular, that $\xi$ is $G_{\infty}$-adapted.

Definition 3.2. A r.v. $\xi$ is said to generate a nontrivial bounded potential if the potential $X_{t}$ in expansion (3.1) can be chosen in such a way that $\mathbb{P}\left\{X_{t}>0\right\}>0$ for any $t>0$.

Definition 3.3. A r.v. $\xi$ is said to predictably generate a bounded potential if the process $A_{t}$ in expansion (3.1) can be chosen to be $G_{t}$-predictable.

Note that for a given $X_{t}$, the predictable process in (3.1) is unique.

Denote $G_{0}$ the family of all $\mathbb{P}$ zero sets of $\mathscr{F}$.

Lemma 3.4. Any integrable r.v. $\xi$ generates a bounded potential.

Proof. Choose any $t_{0}>0$ and set $A_{t}=\xi I\left\{t \geq t_{0}\right\}, G_{t}=\overline{G_{0}} \vee \Omega$ for $t<t_{0}, G_{t}=\mathscr{F}_{\text {for }} t \geq t_{0}$. Then $A_{\infty}=\xi, E\left(A_{\infty}-A_{t} \mid G_{t}\right)=0$ for $t \geq t_{0}$ and $E\left(A_{\infty}-A_{t} \mid G_{t}\right)=E \xi$ for $t<t_{0}$. So, $X_{t}=$ $E \xi I\left\{t<t_{0}\right\}$ is a bounded potential. (Note that $X_{t}$ is trivial in terms of Definition 3.2.)

Theorem 3.5. Let a r.v. $\xi$ satisfy Cramer conditions. Then $\xi$ generates a nontrivial bounded potential.

Proof. According to Theorem 2.7 we can choose some $c>0$ and a sequence $\pi=\left\{t_{n}, n \geq\right.$ $0\}, t_{0}=0, t_{n} \uparrow \infty$ such that $\int_{t_{n}}^{\infty}\left(1-F_{\xi}(u)\right) d u\left(1-F_{\xi}\left(t_{n}\right)\right)^{-1} \leq c$.

Define

$$
\begin{aligned}
G_{t} & := \begin{cases}\overline{G_{0}}, & 0 \leq t<t_{1}, \\
\sigma\left\{\xi \wedge t_{k}, 1 \leq k \leq n\right\}, & t_{n} \leq t<t_{n+1},\end{cases} \\
A_{t} & := \begin{cases}0, & 0 \leq t<t_{1}, \\
\xi \wedge t_{n}, & t_{n} \leq t<t_{n+1},\end{cases}
\end{aligned}
$$

where $n \geq 1$. Then $G_{t}$ satisfies the standard conditions, $A_{t}$ is a $G_{t}$-adapted nonnegative nondecreasing right-continuous process, and $A_{\infty}=\xi$. Now, let $X_{t}:=E\left(A_{\infty}-A_{t} \mid G_{t}\right)$. 
Since for any $k<m$ and $\alpha<t_{k}$, the event $\left\{\xi \wedge t_{k}<\alpha\right\}=\left\{\xi<\alpha \wedge t_{k}\right\}=\left\{\xi \wedge t_{m}<\alpha \wedge t_{k}\right\}$, we have that $G_{t}=\sigma\left\{\xi \wedge t_{n}\right\}$ if $t_{n} \leq t<t_{n+1}$. Therefore, for $t_{n} \leq t<t_{n+1}$,

$$
\begin{aligned}
X_{t} & =E\left(\xi-\xi \wedge t_{n} \mid \xi \wedge t_{n}\right) \\
& =\int_{t_{n}}^{\infty}\left(u-t_{n}\right) d F_{\xi}(u)\left(1-F_{\xi}(u)\right)^{-1} I\left\{\xi>t_{n}\right\} \\
& =\int_{t_{n}}^{\infty}\left(1-F_{\xi}(u)\right) d u\left(1-F_{\xi}(u)\right)^{-1} I\left\{\xi>t_{n}\right\} \leq c I\left\{\xi>t_{n}\right\} \leq c .
\end{aligned}
$$

We conclude that the potential $X_{t}$ is bounded and $\mathbb{P}\left\{X_{t}>0\right\}>\mathbb{P}\left\{\xi>t_{n}\right\}>0, t_{n} \leq t<t_{n+1}$; so $X_{t}$ is nontrivial.

Theorem 3.6. Let a r.v. $\xi$ satisfy the Garsia inequality. Then $\xi$ predictably generates a nontrivial potential.

Proof. Let $G_{t}=\sigma\{\xi \wedge s, s \leq t\}$. Analogously to the proof of Theorem 3.5, we can establish that $G_{t}=\sigma\{\xi \wedge t\}$. Also, let $A_{t}:=\xi \wedge t, A_{\infty}=\xi$. According to Theorem 2.2 and representation (2.1),

$$
X_{t}:=E\left(A_{\infty}-A_{t} \mid G_{t}\right)=E(\xi-\xi \wedge t \mid \xi \wedge t) I\{\xi>t\}=(\lambda(t))^{-1} I\{\xi>t\} \leq c_{0} .
$$

Evidently, $\mathbb{P}\left\{X_{t}>0\right\}>0, t>0$, and $A_{t}$ is continuous. Therefore, it is a predictable process and the proof follows.

Remark 3.7. The $\sigma$-fields $G_{t}$ participating in the proof of Theorem 3.6 were considered by Dellacherie [1], where the dual predictable projection of the process $A=I_{[\xi, \infty[}$ with respect to $\left\{G_{t}, t \geq 0\right\}$ was constructed.

The next example demonstrates that the same r.v. $\xi$ can generate rather different potentials with respect to the same filtration $\left\{G_{t}, t \geq 0\right\}$.

Example 3.8. Let $\left\{P_{t}, t \geq 0\right\}$ be a homogeneous Poisson process with the intensity $\lambda_{0}$, $\theta(x)=\exp \left\{-\lambda_{1} x\right\}, \lambda_{0}, \lambda_{1}>0$. Define $\xi:=\int_{0}^{\infty} \theta(u) P_{u} d u=\lambda_{1}^{-1} \int_{0}^{\infty} \theta(u) d P_{u}$. Then $E \xi=$ $\lambda_{0} \int_{0}^{\infty} u \theta(u) d u=\lambda_{0} \lambda_{1}^{-2}$.

Also, let $G_{t}=\sigma\left\{P_{s}, 0 \leq s \leq t\right\}$. If we put $A_{t}^{0}:=\int_{0}^{t} \theta(u) P_{u} d u, A_{\infty}:=\xi$, then the process $A_{t}$ is predictable, and the potential $X_{t}^{0}:=E\left(A_{\infty}^{0}-A_{t}^{0} \mid G_{t}\right)=P_{t} \int_{t}^{\infty} \theta(u) d u=\lambda_{1}^{-1} \theta(t) P_{t}$ is nontrivial and unbounded for any $t>0$. With $A_{t}^{1}:=\lambda_{1}^{-1} \int_{0}^{t} \theta(u) d P_{u}, A_{\infty}^{1}=\xi, A_{t}^{1}$ is not predictable, and the potential $X_{t}^{1}:=E\left(A_{\infty}^{1}-A_{t}^{1} \mid G_{t}\right)=\lambda_{0} \lambda_{1}^{-1} \int_{t}^{\infty} \theta(u) d u=\lambda_{0} \lambda_{1}^{-1} \theta(t)$ is nontrivial and bounded.

\section{Some additional results}

The next theorem states that in the case when $\overline{\lim }_{x \rightarrow \infty} \lambda(x)>0$, an integrable r.v. $\xi$ can be transformed into a r.v. $\psi(x)$ that predictably generates a bounded potential. Note that in this case we can choose the sequence $\pi=\left\{t_{n}, n \geq 0\right\}, t_{0}=0, t_{n} \uparrow \infty$ such that

$$
\lambda\left(t_{n}\right) \geq c>0, \quad \delta_{\pi}:=\inf _{n \geq 1}\left(t_{n}-t_{n-1}\right)>0 .
$$


Theorem 4.1. Let $\varlimsup_{x \rightarrow \infty} \lambda(x)>0$, let $\pi\left\{t_{n}, n \geq 0\right\}$ be chosen as in (4.1). Denote by $\psi$ : $\mathbb{R}_{+} \rightarrow \mathbb{R}_{+}$an increasing piece-wise linear function such that $\psi\left(t_{n}\right)=n$.

Then $\psi(\xi)$ predictably generates a nontrivial bounded potential.

Proof. The d.f. of the r.v. $\psi(\xi)$ equals $F_{\psi}(x)=\mathbb{P}\{\psi(\xi) \leq x\}=\mathbb{P}\left\{\xi \leq \psi^{-1}(x)\right\}$. Therefore,

$$
\begin{aligned}
\int_{n}^{\infty}\left(1-F_{\psi}(u)\right) d u \cdot\left(1-F_{\psi}(n)\right)^{-1} \\
=\int_{n}^{\infty}\left(1-F_{\xi}\left(\psi^{-1}(u)\right)\right) d u \cdot\left(1-F_{\xi}\left(t_{n}\right)\right)^{-1} \\
=\int_{t_{n}}^{\infty}\left(1-F_{\xi}(u)\right) \psi^{\prime}(u) d u \cdot\left(1-F_{\xi}\left(t_{n}\right)\right)^{-1} .
\end{aligned}
$$

Furthermore, for $t \in\left(t_{n-1}, t_{n}\right)$,

$$
\psi^{\prime}(t)=\left(\psi\left(t_{n}\right)-\psi\left(t_{n-1}\right)\right)\left(t_{n}-t_{n-1}\right)^{-1}=\left(t_{n}-t_{n-1}\right)^{-1} \leq \delta_{\pi}^{-1}
$$

We obtain from (4.2) and (4.3) that there exists $c_{1}>0$ such that $\left(\lambda_{\psi}(n)\right)^{-1}:=\int_{n}^{\infty}(1-$ $\left.F_{\psi}(u)\right) d u\left(1-F_{\psi}(n)\right)^{-1} \leq c_{1}$. Note that $x\left(1-F_{\psi}(x)\right)=x\left(1-F_{\xi}\left(\psi^{-1}(x)\right)\right)=\psi^{-1}(x)(1-$ $\left.F_{\xi}\left(\psi^{-1}(x)\right)\right) \cdot\left(x\left(\psi^{-1}(x)\right)^{-1}\right)$, and $\psi^{-1}(x)\left(1-F_{\xi}\left(\psi^{-1}(x)\right)\right) \rightarrow 0, x \rightarrow \infty, x\left(\psi^{-1}(x)\right)^{-1} \leq$ $(n+1) / \psi^{-1}(n)=(n+1) / t_{n}<(n+1) / \delta_{\pi} n \leq c_{2}, n<t \leq n+1$.

It means that $\int_{0}^{\infty} u d F_{\psi}(u)=\int_{0}^{\infty}\left(1-F_{\psi}(u)\right) d u<\infty$, that is, $\psi$ is integrable.

Now, let $G_{t}=\sigma\{\psi(\xi) \wedge n\}, n \leq t<n+1$, and

$$
A_{t}:= \begin{cases}0, & 0 \leq t<2 \\ \psi(\xi) \wedge(n-1), & n \leq t<n+1\end{cases}
$$

Then $A_{t}$ is $G_{t}$-predictable, $A_{\infty}=\psi(\xi)$, and for any $t>0$,

$$
\begin{aligned}
X_{t} & :=E\left(A_{\infty}-A_{t} \mid G_{t}\right) \\
& =E(\psi(\xi)-\psi(\xi) \wedge n \mid \psi(\xi) \wedge n)+(\psi(\xi) \wedge n-\psi(\xi) \wedge(n-1)) \leq c_{1}+1 .
\end{aligned}
$$

The next result demonstrates that an integrable r.v. $\xi$ with $\varlimsup_{x \rightarrow \infty} \lambda(x)>0$ generates an "almost bounded" potential.

Theorem 4.2. Let $\varlimsup_{x \rightarrow \infty} \lambda(x)>0$. Then for any $\varepsilon>0$, the r.v. $\xi$ predictably generates a nontrivial potential that is bounded on the set $\mathbb{R}_{+} \backslash B$ where $m(B)<\varepsilon(m(\cdot)$ is a Lebesgue measure on $\mathbb{R}$ ).

Proof. Consider $G_{t}:=\sigma\left\{\xi \wedge t_{n}\right\}, t_{n}-\varepsilon / 2^{n} \leq t<t_{n+1}-\varepsilon / 2^{n+1}$, and $A_{t}$ being the same as in the proof of Theorem 3.5. Then $A_{t}$ is $G_{t}$-predictable. Furthermore, $X_{t}:=E\left(A_{\infty}-\right.$ $\left.A_{t} \mid G_{t}\right)=E\left(\xi-\xi \wedge t_{n} \mid \xi \wedge t_{n}\right) \leq c$ for $t_{n} \leq t<t_{n+1}-\varepsilon / 2^{n+1}$. 
Remark 4.3. Let the r.v. $\xi$ be such that $0=\underline{\lim }_{x \rightarrow \infty} \lambda(x)<\varlimsup_{x \rightarrow \infty} \lambda(x)$. Consider $G_{t}$ and $A_{t}$ from the proof of Theorem 3.5 and construct the compensator (dual predictable projection) $A_{t}^{\pi}$ of $A_{t}$. Evidently,

$$
\begin{aligned}
A_{t}^{\pi} & =\sum_{k=1}^{n} E\left(\xi \wedge t_{k}-\xi \wedge t_{k-1} \mid \xi \wedge t_{k-1}\right) \\
& =\sum_{k=1}^{n} \int_{t_{n-1}}^{t_{n}}\left(x-t_{n-1}\right) d F_{\xi}(x)\left(1-F_{\xi}\left(t_{n-1}\right)\right)^{-1} I\left\{\xi>t_{n-1}\right\}, \quad t_{n} \leq t<t_{n+1}, \\
A_{\infty}^{\pi} & =\sum_{k=1}^{n} \int_{t_{n-1}}^{t_{n}}\left(x-t_{n-1}\right) d F_{\xi}(x)\left(1-F_{\xi}\left(t_{n-1}\right)\right)^{-1} I\left\{\xi>t_{n-1}\right\} .
\end{aligned}
$$

Since $A_{t}-A_{t}^{\pi}$ is a $G_{t}$-martingale, we obtain that

$$
X_{t}:=E\left(A_{\infty}-A_{t} \mid G_{t}\right)=E\left(A_{\infty}^{\pi}-A_{t}^{\pi} \mid G_{t}\right) \leq c .
$$

The last relation means that $A_{\infty}^{\pi}$ satisfies the Garsia inequality.

\section{Acknowledgment}

This research is partially supported by INTAS 99-00016.

\section{References}

[1] C. Dellacherie, Capacités et Processus Stochastiques, Springer-Verlag, Berlin, 1972.

[2] A. M. Garsia, Martingale Inequalities: Seminar Notes on Recent Progress, W. A. Benjamin, Reading, Massachusetts, 1973.

[3] B. Ramachandran, Advanced Theory of Characteristic Functions, Statistical Publishing Society, Calcutta, 1967.

N. Kartashov: Department of Mathematics, Kiev National University, 64 Vladimirskaya Street, 01033 Kiev, Ukraine

E-mail address: winf@carrier.kiev.ua

Yu. Mishura: Department of Mathematics, Kiev National University, 64 Vladimirskaya Street, 01033 Kiev, Ukraine

E-mail address: myus@univ.kiev.ua 


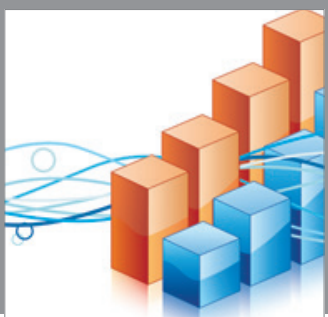

Advances in

Operations Research

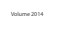

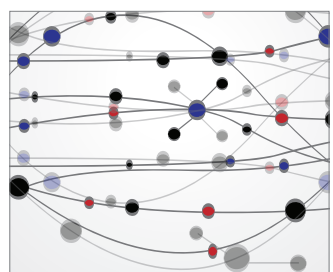

\section{The Scientific} World Journal
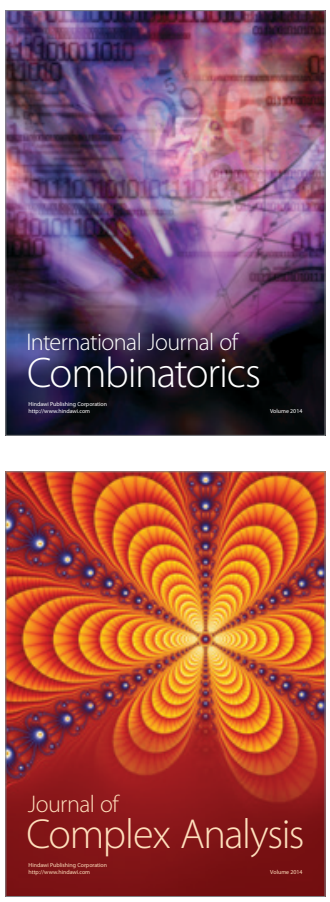

International Journal of

Mathematics and

Mathematical

Sciences
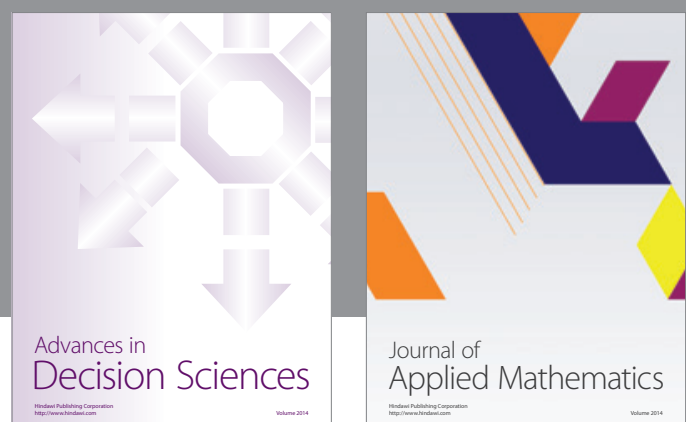

Journal of

Applied Mathematics
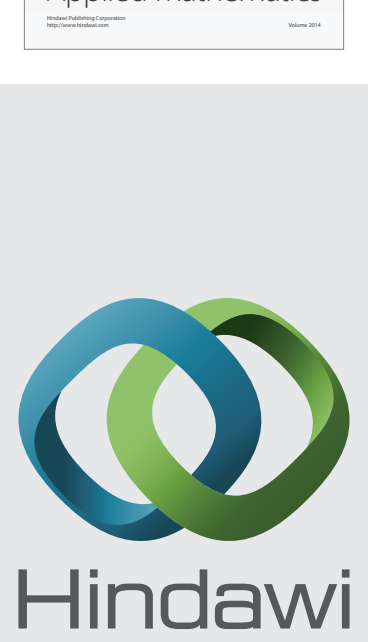

Submit your manuscripts at http://www.hindawi.com
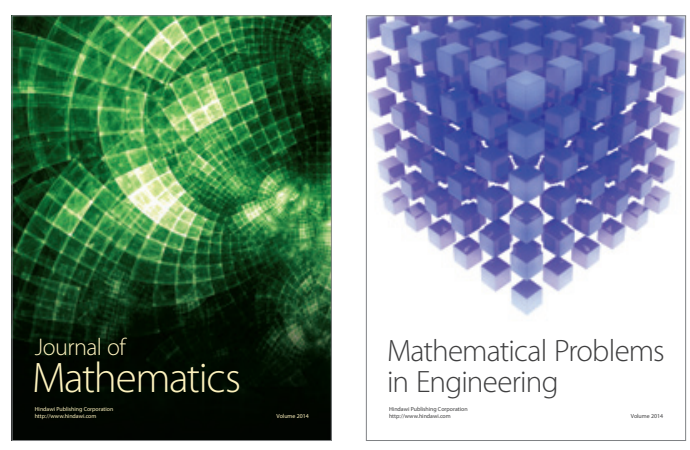

Mathematical Problems in Engineering
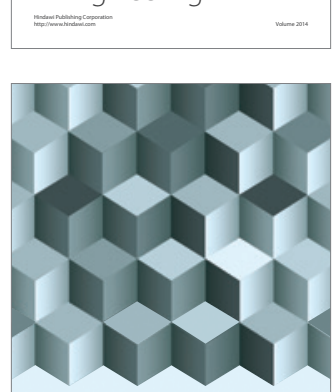

Journal of

Function Spaces
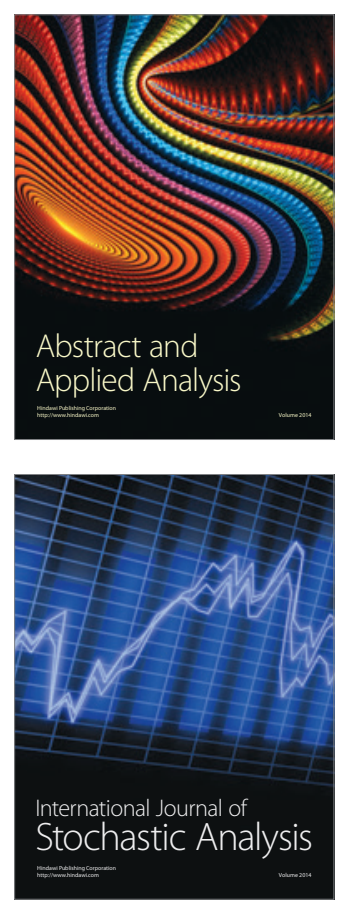

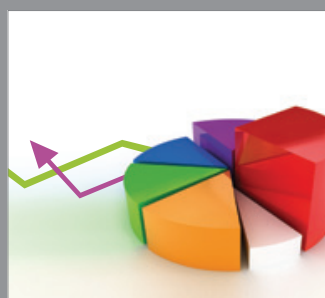

ournal of

Probability and Statistics

Promensencen
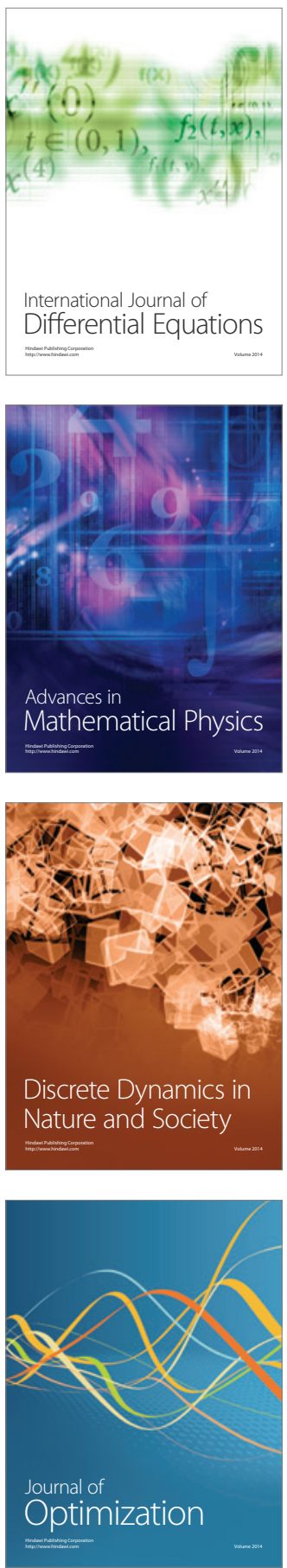